PROCEEDINGS OF THE

AMERICAN MATHEMATICAL SOCIETY

Volume 138, Number 3, March 2010, Pages 1085-1092

S 0002-9939(09)10095-3

Article electronically published on October 23, 2009

\title{
GEODESICS AVOIDING SUBSETS IN HADAMARD MANIFOLDS
}

\author{
ALBERT BORBÉLY
}

(Communicated by Jon G. Wolfson)

\begin{abstract}
Let $M^{n}, n \geq 3$, be an $s$-hyperbolic (in the sense of Gromov) Hadamard manifold. Let us assume that we are given a family of disjoint convex subsets and a point $o$ outside these sets. It is shown that if one shrinks these sets by the constant $s$, then it is possible to find a complete geodesic through $o$ that avoids the shrunk sets.
\end{abstract}

\section{INTRODUCTION}

The problem of finding geodesics or geodesic rays that avoid certain obstacles in negatively curved manifolds has been studied in different situations. It found applications in several areas like the existence of bounded geodesics in manifolds with finite volume [S], PP1], [PP2] and the construction of proper closed invariant subsets of the unit tangent tangent bundle with large footprints [BSW], [W].

In the papers of $[\mathrm{S}],[\mathrm{BSW}]$ and $[\mathrm{W}]$ the following problem was considered. Given an $a$-separated set (the distance between any two points is at least $a>0$ ) find a geodesic ray or geodesic that avoids these points by a distance of $\epsilon>0$. Various conditions were given for the existence and non-existence along with some applications. In [PP1] and [PP2] instead of an $a$-separated set a family of disjoint balls and horoballs were given, and after shrinking them by a fixed constant one had to find a geodesic ray avoiding the shrunk balls. The two problems are related in an obvious way.

In these examples the obstacles were always regular: balls or horoballs. In a previous paper $[\mathrm{B}$ ] we extended some of the results involving balls and horoballs for arbitrary convex sets. However our method produced only geodesic rays, which are not entirely satisfactory, since for the applications given in $[\mathrm{S}$ ] one needs complete geodesics.

Based on the results of $[\mathrm{BSW}$ ] and $[\mathrm{S}$ ] it seems that producing geodesics that avoid $a$-separated sets by a distance of $\epsilon$ is considerably harder - and in some cases impossible - than producing geodesic rays. In this paper we improve the results of B] to obtain geodesics instead of geodesic rays. This could be used in place of [S, Proposition 1] in the applications given.

There is another improvement, however. The main technical ingredient in $[\mathrm{B}]$ is a simple lemma (Lemma 1) that was also used in the original version of this paper. It was the referee who observed that the statement of that lemma is exactly the

Received by the editors June 12, 2008.

2000 Mathematics Subject Classification. Primary 53C22.

Key words and phrases. Convex sets, negative curvature, geodesics.

(C)2009 American Mathematical Society Reverts to public domain 28 years from publication 
same as the Rips definition of Gromov hyperbolicity. This means that instead of putting an upper bound on the curvature (say $K \leq-1$ ), it is enough to require the manifold to be Gromov hyperbolic.

Definition. For a Hadamard manifold $M^{n}$ we say it is $s$-hyperbolic $(s>0)$ if the following holds: given a geodesic triangle $\triangle_{p q r}$ and a point $x \in[p, q]$ if $B(x, s) \cap$ $[p, r]=\emptyset$, then the geodesic segment $[r, q]$ intersects $B(x, s)$.

It is easy to see that this is equivalent to the statement that the geodesic segment $[p, q]$ is in the $s$-neighborhood of the segments $[p, r] \cup[r, q]$, which is the Rips definition of Gromov hyperbolicity; see for example the first chapter of [BS].

It is known that a Hadamard manifold with $K<-1$ is $s$-hyperbolic for some $s>0$. The best value can be computed from the ideal triangle in the hyperbolic plane, and it is $s=\frac{1}{2} \ln (\sqrt{2}+1) \approx 0.44 \ldots$. This value is different from the one given in [BS], exercise 1.4.1, because our definition of $s$-hyperbolicity is different.

Let $M^{n}$ be an $n$-dimensional $(n \geq 3)$ Hadamard manifold that is a complete and simply connected Riemannian manifold with sectional curvatures $\leq 0$. For a convex set $C \subset M^{n}$ and a positive number $t>0$ we define the $t$-kernel of $C$ as

$$
C(t)=\{x \in C: \operatorname{dist}(x, \partial C)>t\} .
$$

It is easy to see that the $t$-kernel is always open, possibly empty. Our result can now be stated as follows.

Theorem. Let $M^{n}, n \geq 3$, be an s-hyperbolic Hadamard manifold and $C_{n} \subset$ $M, \quad n=1,2, \ldots$, be any family of disjoint open convex sets. Then for any point $o \in M-\bigcup C_{n}$ there is a complete geodesic through o avoiding the shrank sets $C_{n}(s)$ for all $n$.

The statement clearly fails for surfaces. Even for $\mathbb{H}^{2}(-1)$ one can easily find a counterexample. Consider three half-spaces with viewing-angle $120^{0}$ viewed from $o$. Then, regardless of how much we shrink them, there are exactly three geodesic rays avoiding these shrank half-spaces, but every geodesic through $o$ will intersect one of them.

\section{Preliminaries}

For the rest of the paper let $s>0$ be a fixed positive number, $M$ be an $n$ dimensional $(n \geq 3) s$-hyperbolic Hadamard manifold, $C_{n} \subset M, \quad n=1,2, \ldots$, be a family of disjoint open convex sets and $o \in M-\bigcup C_{n}$ be an arbitrary point.

Denote by $S$ the sphere at infinity (viewed as the set of equivalence classes of geodesic rays) and identify it with the unit sphere of the tangent space $T_{o} M$ in the obvious way.

Define the projection maps $\pi: M \cup S-\{o\} \rightarrow S$ and $-\pi: M \cup S-\{o\} \rightarrow S$ respectively such that $\pi(q)=\left[\gamma_{o q}\right]$ and $-\pi(q)=\left[\gamma_{q o}\right]$ resp., where $\gamma_{x y}$ denotes the geodesic ray starting from $x$ and passing through $y$, and for a geodesic ray $\gamma,[\gamma] \in S$ denotes the equivalence class. This way $\pi(q)$ and $-\pi(q)$ are "mirror" images with respect to the "center" $o \in M$.

The following is now immediate.

Proposition 1.1. If $D \subset M-\{o\}$ is open, then $\pi(D)$ and $-\pi(D)$ are open sets in $S$, and if $F \subset M$ is closed and bounded, then $\pi(F)$ and $-\pi(F)$ are closed in $S$. 
The proof is obvious.

It will be convenient to introduce the following relations between subsets of $M-\{o\}$. Let $F \subset M-\{o\}$ be any set and $x \in M-\{o\}$ be any point. We say that $x$ is "below" $F$ (viewed from the point $o$ ) if $[o x] \cap F=\gamma_{o x} \cap F \neq \emptyset$, where [ox] denotes the geodesic segment connecting $o$ and $x$ and $\gamma_{o x}$ denotes the geodesic ray emanating from $o$ and passing through $x$.

The following definitions will be useful.

Definition 1.2. For two sets $A, B \subset M \cup S-\{o\}$ we say that $A>B$ if every point of $B$ is below $A$ in the above sense.

It is clear that this relation is transitive.

Definition 1.3. For two sets $A, B \subset M \cup S-\{o\}$ we say that $A \rightarrow B$ if there are points $a \in A(s)$ and $b \in B$ such that $b$ is below $a$ in the above sense.

Although the relation $\rightarrow$ is not transitive for disjoint convex sets, $A \rightarrow B$ implies $A>B$.

Proposition 1.4. If $A, B \subset M-\{o\}$ are disjoint open convex sets such that $A \rightarrow B$, then $A>B$.

Proof. Since $A \rightarrow B$ there are according to Definition 1.3 points $a \in A(s)$ and $b \in B$ such that $a \in[o b]$. Let $b^{\prime} \in B$ be an arbitrary point.

If the segment $\left[o b^{\prime}\right]$ does not intersect $A$, then it will not intersect the geodesic ball $B(a, s) \subset A$, and according to the definition of $s$-hyperbolicity the geodesic segment $\left[b^{\prime} b\right]$ must intersect $B(a, s)$. Since $B$ is convex this implies that $A \cap B \neq \emptyset$, and that is a contradiction.

Therefore $\left[o b^{\prime}\right]$ intersects $A$, and since $A$ is convex and disjoint from $B$ we have that $b^{\prime}$ is below $A$. Taking into consideration that $b^{\prime}$ was arbitrary we obtain $A>B$.

The main technical ingredient in the proof of the Theorem is the following.

Lemma 1.5. For every connected component $D$ of $\bigcup_{n=1}^{m} \pi\left(C_{n}(s)\right)$ there is a $C_{i}$ such that $D \subset \pi\left(C_{i}\right)$.

Since $C_{i}$ is convex, $\pi\left(C_{i}\right)$ is contained in a half-sphere of the sphere at infinity $S$. This shows that $D \neq S$; therefore $S \neq \bigcup_{n=1}^{\infty} \pi\left(C_{n}(s)\right)$, which immediately implies the existence of a geodesic ray emanating from $o$ and avoiding all the sets $C_{n}(s)$, $n=1,2, \ldots$. However, this time we have to prove more: the existence of a complete geodesic through $o$ avoiding all the sets $C_{n}(s), n=1,2, \ldots$.

\section{Proof of Lemma 1.5}

In this section we will prove a more general statement about the structure of the sets $\left\{C_{n}\right\}_{n=1}^{\infty}$. The main technical ingredient of this section is the following.

Proposition 2.1. Let $A, B, C \subset M-\{o\}$ be disjoint open and convex sets such that $A \rightarrow B$ and $C \rightarrow B$. Then either $C \rightarrow A$ or $A \rightarrow C$.

Proof. From the assumptions it follows that there are points $a \in A(s), b, b^{\prime} \in B$ and $c \in C(s)$ such that $a \in[o, b]$ and $c \in\left[o, b^{\prime}\right]$. Moreover from Proposition 1.4 we obtain that $A>B$ and $C>B$. This implies that for every $x \in\left[b^{\prime}, b\right]$ the sets $A_{x}=A \cap[o, x] \neq \emptyset$ and $C_{x}=C \cap[o, x] \neq \emptyset$ are not empty. 
Taking into account that $A, C$ are disjoint open and convex sets, we know that $A_{x}$ and $C_{x}$ are disjoint open segments of $[o, x]$. Therefore either $A_{x}$ is below $C_{x}$ or $C_{x}$ is below $A_{x}$. Define $G_{A}$ and $G_{C}$ as follows:

$$
G_{A}=\left\{x \in\left[b^{\prime}, b\right]: \quad C_{x} \text { below } A_{x}\right\}
$$

and

$$
G_{C}=\left\{x \in\left[b^{\prime}, b\right]: \quad A_{x} \text { below } C_{x}\right\} .
$$

Then clearly $G_{A}$ and $G_{C}$ are disjoint open subsets of $\left[b^{\prime}, b\right]$ such that $G_{A} \cup G_{c}=$ $\left[b^{\prime}, b\right]$. Since $\left[b^{\prime}, b\right]$ is connected we obtain that either $G_{A}=\emptyset$ or $G_{C}=\emptyset$.

If $G_{A}=\emptyset$, then $b^{\prime} \in G_{C}$, which implies that $C \rightarrow A$, and similarly $G_{C}=\emptyset$ implies $A \rightarrow C$.

To state our next lemma it will be convenient to consider the directed graph $\Gamma\left\{\left\{C_{n}\right\}_{n=1}^{\infty}, \rightarrow\right\}$. We call a subset $F \subset\left\{C_{n}\right\}_{n=1}^{\infty}$ an undirected connected component if for every two sets $A, B \in F$ there is an undirected path in $\Gamma$ connecting them, that is, a sequence of sets $C_{1}=A, C_{2}, \ldots, C_{n}=B$ such that either $C_{i} \rightarrow C_{i+1}$ or $C_{i+1} \rightarrow C_{i}$. The following statement will imply Lemma 1.5.

Lemma 2.2. Let $F \subset\left\{C_{n}\right\}_{n=1}^{\infty}$ be a finite undirected connected component of $\Gamma$. Then there is a unique set (the top set) $A \in F$ such that for every $B \in F$ there is a directed path in $\Gamma$ connecting $A$ to $B$.

Proof of Lemma 2.2. It is easy to see that the top set is unique. Otherwise $F$ would contain a directed circle containing the set $A$, and from Proposition 1.4 and the transitivity of the relation " $>$ " we would obtain that $A>A$, which is clearly impossible.

The proof proceeds by induction on the number of sets. If $F$ has one or two elements the statement is obviously true. Let us assume that $F=\left\{C_{1}, \ldots, C_{m}\right\}$ and that the statement is true if the number of sets in an undirected component is less than $m$.

Since from every finite connected graph one can remove a vertex such that the remaining graph remains connected, we may assume without loss of generality that the subset $F^{\prime}=\left\{C_{2}, \ldots, C_{m}\right\}$ is an undirected connected component of $\Gamma$ and by induction it has a top set. By renumbering the sets if necessary we may assume that $C_{2}$ is the top set of $F^{\prime}$; that is, there is a directed path from $C_{2}$ to every element of $F^{\prime}$. It will be shown that either $C_{1}$ or $C_{2}$ is the top set of $F$. For this it is enough to show that either $C_{1} \rightarrow C_{2}$ or there is a directed path from $C_{2}$ to $C_{1}$.

Since $F$ is connected there is a $C_{i} \in F^{\prime}$ such that either $C_{i} \rightarrow C_{1}$ or $C_{1} \rightarrow C_{i}$. If $C_{i} \rightarrow C_{1}$, then we are done, since there is a directed path from $C_{2}$ to $C_{i}$ and therefore to $C_{1}$.

In case $C_{1} \rightarrow C_{i}$ we argue as follows. Since there is a directed path from $C_{2}$ to $C_{i}$ by renumbering the sets if necessary, we may as well assume that $C_{2} \rightarrow C_{3}$, $C_{3} \rightarrow C_{4}, \ldots, C_{i-1} \rightarrow C_{i}$. Applying Proposition 2.1 to the sets $C_{i-1}, C_{i}$ and $C_{1}$ we obtain that either $C_{1} \rightarrow C_{i-1}$ or $C_{i-1} \rightarrow C_{1}$. If $C_{i-1} \rightarrow C_{1}$, then we are done as before. In case $C_{1} \rightarrow C_{i-1}$ we repeat the previous argument. This way one obtains that there is a set $C_{j}, 2 \leq j \leq i$ such that either $C_{j} \rightarrow C_{1}$ or $C_{1} \rightarrow C_{2}$.

If $C_{j} \rightarrow C_{1}$, then we argue as before. If $C_{1} \rightarrow C_{2}$, then there is directed path from $C_{1}$ to every element of $F$, and the proof is complete.

We finish the section by showing how Lemma 2.2 implies Lemma 1.5. 
Proof of Lemma 1.5. Let $D \subset S$ be a connected component of $\bigcup_{n=1}^{m} \pi\left(C_{n}(s)\right)$. By discarding sets if necessary, without loss of generality we may assume that $C_{n}(s) \neq \emptyset$ for $n=1, \ldots, m$ and $D=\bigcup_{n=1}^{m} \pi\left(C_{n}(s)\right)$. Since $D$ is connected it is easy to see that for every $i, j \in\{1, \ldots, m\}$ there is a chain of sets $C_{i}=C_{i_{1}}, \ldots, C_{i_{k}}=C_{j}$ such that $\pi\left(C_{i_{l}}(s)\right) \cap \pi\left(C_{i_{l+1}}(s)\right) \neq \emptyset$. According to Definition 1.3

$$
\pi\left(C_{i_{l}}(s)\right) \cap \pi\left(C_{i_{l+1}}(s)\right) \neq \emptyset
$$

implies that either $C_{i_{l}} \rightarrow C_{i_{l+1}}$ or $C_{i_{l+1}} \rightarrow C_{i_{l}}$. This means that there is an undirected path connecting $C_{i}$ to $C_{j}$; that is, $F=\left\{C_{n}\right\}_{n=1}^{m}$ is an undirected connected component of $\Gamma$.

Applying Lemma 2.2 we conclude that $F$ has a top set which we may assume to be $C_{1}$. This means that for every $C_{i}$ there is a directed path connecting $C_{1}$ to $C_{i}$. Applying Proposition 1.4 repeatedly we obtain that $C_{1}>C_{i}$ for $i=2, \ldots, m$. Therefore $\pi\left(C_{1}\right) \supset \bigcup_{1=1}^{m} \pi\left(C_{i}\right) \supset D$, and this concludes the proof of Lemma 1.5.

\section{Proof of the Theorem}

The proof of the Theorem relies on Lemma 1.5 and the following proposition.

Proposition 3.1. Let $D_{i} \subset S^{n}(n \geq 2), i=1,2, \ldots$, be a finite or infinite family of pairwise disjoint open sets such that for every $D_{i}$ there is an open half-sphere $S_{i}$ with $D_{i} \subset S_{i}$. Then $S^{n} \neq\left(\cup D_{i}\right) \cup\left(\cup-D_{i}\right)$, where $-D_{i}$ denotes the antipodal image of $D_{i}$.

The statement fails to be true for $n=1$. One can take as a simple counterexample three equal consecutive arcs of the unit circle. For $n>1$ it follows from the following statement.

Proposition 3.2. Let $U_{i} \subset S^{n}, i \in I$, be an open covering of $S^{n}$ such that every $U_{i}$ is contained in an open hemisphere. Then there is a point $x \in S^{n}$ which is contained in at least $(n+1)$ covering sets.

The statement of Proposition 3.2 is known, and one can find it in [BO], [LS] or in $[\mathrm{M}$ ] for a recent reference. However, for the convenience of the reader we provide a short proof in the next section.

Proof of Proposition 3.1. We argue indirectly. Suppose $S^{n}=\left(\cup D_{i}\right) \cup\left(\cup-D_{i}\right)$. According to Proposition 3.2 there must be three sets with non-empty intersection. But two of these sets must belong to the same family of sets (either $\left\{D_{i}\right\}_{i \in I}$ or $\left.\left\{-D_{i}\right\}_{i \in I}\right)$, which contradicts the assumption that $\left\{D_{i}\right\}_{i \in I}$ and $\left\{-D_{i}\right\}_{i \in I}$ are families of pairwise disjoint sets.

Proof of the Theorem. To show the existence of a geodesic through $o$ that avoids the sets $C_{n}(s)$ for all $n$, it is enough to show that

$$
S \neq\left(\bigcup_{n=1}^{\infty} \pi\left(C_{n}(s)\right)\right) \cup\left(\bigcup_{n=1}^{\infty}-\pi\left(C_{n}(s)\right)\right) .
$$

We argue indirectly. Let us assume that

$$
S=\left(\bigcup_{n=1}^{\infty} \pi\left(C_{n}(s)\right)\right) \cup\left(\bigcup_{n=1}^{\infty}-\pi\left(C_{n}(s)\right)\right) .
$$


Since $S$ is compact we have

$$
S=\left(\bigcup_{n=1}^{m} \pi\left(C_{n}(s)\right)\right) \cup\left(\bigcup_{n=1}^{m}-\pi\left(C_{n}(s)\right)\right)
$$

for some $m \in \mathbb{N}$.

Denote the connected components of $\bigcup_{n=1}^{m} \pi\left(C_{n}(s)\right)$ by $D_{1}, D_{2}, \ldots$. By Lemma 1.5 for every $D_{i}$ there is a $C_{j}$ such that $D_{i} \subset \pi\left(C_{j}\right)$. Since $C_{j}$ is convex $\pi\left(C_{j}\right)$ is contained in a half-sphere of the sphere at infinity $S$. Therefore every $D_{i}$ is contained in a half-sphere, and since by definition the sets are pairwise disjoint from Proposition 3.1, we obtain that

$$
S \neq\left(\bigcup D_{n}\right) \cup\left(\bigcup-D_{n}\right) .
$$

Since

$$
\bigcup D_{n}=\bigcup_{n=1}^{m} \pi\left(C_{n}(s)\right) \text { and } \bigcup-D_{n}=\bigcup_{n=1}^{m}-\pi\left(C_{n}(s)\right),
$$

we get a contradiction to (3.3). This proves (3.2) and the Theorem as well.

\section{Proof of Proposition 3.2}

For every $U_{i}$ pick an open hemisphere $G_{i}$ such that $U_{i} \subset G_{i}$. Since the hemispheres of $S^{n}$ are convex, the cover $\left\{G_{i}\right\}_{i \in I}$ is a "good cover", which means that all non-empty intersections are contractible. At the same time the cover $\left\{U_{i}\right\}_{i \in I}$ is a refinement of $\left\{G_{i}\right\}_{i \in I}$.

To prove Proposition 3.2 basically we have to show that any open cover of $S^{n}$ which is a refinement of a "good cover" has covering dimension greater than or equal to $n$.

We argue indirectly. Suppose Proposition 3.2 fails for the cover $\left\{U_{i}\right\}_{i \in I}$. This means that the $(n+1)$-fold intersections of the covering sets are empty; that is, the covering dimension is less than $n$. From the definition of the Čech cohomology one obtains that $\check{H}^{n}(U, \mathbb{R})=0$, where $\check{H}^{n}(U, \mathbb{R})$ denotes the $n$th cohomology of the cover $\left\{U_{i}\right\}_{i \in I}$ with values in $\mathbb{R}$.

On the other hand since $\left\{G_{i}\right\}_{i \in I}$ is a "good cover", we know that the Cech cohomology of the cover is isomorphic to the de Rham cohomology of $S^{n}$ (Theorem 8.9 in [BT] $)$. Therefore $\check{H}^{n}(G, \mathbb{R})=\mathbb{R}$, where $\check{H}^{n}(G, \mathbb{R})$ denotes the $n$th cohomology of the cover $\left\{G_{i}\right\}_{i \in I}$ with values in $\mathbb{R}$. This would facilitate the desired contradiction provided that the map

$$
\check{H}^{n}(G, \mathbb{R}) \rightarrow \check{H}^{n}(U, \mathbb{R})
$$

induced by the restriction is not trivial. For the lack of an exact reference to this fact we prove a different statement that provides the required contradiction equally well. The following statement implies Proposition 3.2 in an obvious way.

Proposition 4.1. Let $X^{n}$ be an $n$ dimensional $n>0$ manifold, $\left\{G_{i}\right\}_{i \in I}$ be a "good cover" and $\left\{U_{j}\right\}_{j \in J}$ be an open cover which is a refinement of the cover $\left\{G_{i}\right\}_{i \in I}$. If the $(n+1)$-fold intersections of the cover $\left\{U_{j}\right\}_{j \in J}$ are empty, then the nth de Rham cohomology group of $X$ vanishes; that is, $H_{d}^{n}(X, \mathbb{R})=0$.

All that remains is to prove this last proposition. 
Proof of Proposition 4.1. The proof is a diagram chase. As in [BT consider the following double complex:

$$
\begin{array}{cccccccc}
\uparrow d & \uparrow d & & \uparrow d & & \uparrow d & & \uparrow d \\
0 \rightarrow \Omega^{2}(X) \stackrel{r}{\rightarrow} & K_{G}^{0,2} & \stackrel{\delta}{\rightarrow} & K_{G}^{1,2} & \stackrel{\delta}{\rightarrow} & K_{G}^{2,2} & \stackrel{\delta}{\rightarrow} & K_{G}^{3,2} \stackrel{\delta}{\rightarrow} \\
\uparrow d & \uparrow d & & \uparrow d & & \uparrow d & & \uparrow d \\
0 \rightarrow \Omega^{1}(X) \stackrel{r}{\rightarrow} & K_{G}^{0,1} & \stackrel{\delta}{\rightarrow} & K_{G}^{1,1} & \stackrel{\delta}{\rightarrow} & K_{G}^{2,1} & \stackrel{\delta}{\rightarrow} & K_{G}^{3,1} \stackrel{\delta}{\rightarrow} \\
\uparrow d & \uparrow d & & \uparrow d & & \uparrow d & & \uparrow d \\
0 \rightarrow \Omega^{0}(X) \stackrel{r}{\rightarrow} & K_{G}^{0,0} & \stackrel{\delta}{\rightarrow} & K_{G}^{1,0} & \stackrel{\delta}{\rightarrow} & K_{G}^{2,0} & \stackrel{\delta}{\rightarrow} & K_{G}^{3,0} \\
& \stackrel{\delta}{\rightarrow}
\end{array}
$$

where $\Omega^{q}(X)$ is the set of $q$-forms on $X$ and $K_{G}^{p, q}$ consists of the $p$-cochains of the cover $\left\{G_{i}\right\}_{i \in I}$ with values in the $q$-forms; that is, the elements of $K_{G}^{p, q}$ are $q$-forms defined on the $(p+1)$-fold intersections. The horizontal map $r$ is the restriction map and $\delta$ is the difference operator. The vertical maps $d$ are the exterior derivatives. Moreover the operators $d$ and $\delta$ commute; that is, $d \delta=\delta d$.

Due to the existence of a partition of unity subordinate to $\left\{G_{i}\right\}_{i \in I}$, the horizontal rows (the generalized Mayer-Vietoris sequences) are exact (Proposition 8.5 of BT] $)$. Since all non-empty $(p+1)$-fold intersections of $\left\{G_{i}\right\}_{i \in I}$ are contractible, the columns (with the exception of the first column) are also exact. This makes the following construction possible.

Let $w^{n} \in \Omega^{n}(X)$ be an $n$-form. Since it is a top form, it is closed. We need to show that it is exact. Starting from $w^{n}$ we move in a zig-zag fashion (one step to the right and one step down) through the above diagram. After $2 k$ steps we arrive at $\beta_{k-1}^{n-k} \in K_{G}^{k-1, n-k}$, and after $2 k+1$ steps we have $\alpha_{k}^{n-k} \in K_{G}^{k, n-k}$.

The following statements can be verified inductively. All the $k$-cochains $\alpha_{k}^{n-k}$ are closed. Since the columns are exact this gives rise to the $k$-cochains $\beta_{k}^{n-k-1}$ such that $d \beta_{k}^{n-k-1}=\alpha_{k}^{n-k}$, where $\alpha_{k+1}^{n-k-1}=\delta \beta_{k}^{n-k-1}$. Eventually we get the $(n-1)$-cochain $\beta_{n-1}^{0} \in K_{G}^{n-1,0}$.

Fix a map $\phi: J \rightarrow I$ such that $U_{i} \subset G_{\phi(i)}$. This induces a map

$$
\phi^{\sharp}: K_{G}^{p, q} \rightarrow K_{U}^{p, q}
$$

by restriction.

Now consider the cochains $\phi^{\sharp}\left(\alpha_{k}^{n-k}\right)$ and $\phi^{\sharp}\left(\beta_{k-1}^{n-k}\right)$. Since the rest of the argument takes place in the double complex where the sets $K_{G}^{p, q}$ are replaced with $K_{U}^{p, q}$, for simplicity we will write $\beta_{k-1}^{n-k}$ instead of $\phi^{\sharp}\left(\beta_{k-1}^{n-k}\right)$ and $\alpha_{k}^{n-k}$ instead of $\phi^{\sharp}\left(\alpha_{k}^{n-k}\right)$.

Starting from $\beta_{n-1}^{0} \in K_{U}^{n-1,0}$ we will move in a zig-zag fashion (this time one step left and one step up) through the double complex $\left\{\Omega^{q}, K_{U}^{p, q}\right\}$ to $\Omega^{n-1}(X)$. We can make the horizontal moves due to the exactness of the rows (Proposition 8.5 of BT). The details are as follows.

By the assumption the $(n+1)$-fold intersections of the cover $\left\{U_{j}\right\}_{j \in J}$ are empty; therefore $K_{U}^{n, *}=0$. This means that $\delta\left(\beta_{n-1}^{0}\right)=0$, and from the exactness of the row we obtain an element $\gamma_{n-2}^{0} \in K_{U}^{n-2,0}$ such that

$$
\delta\left(\gamma_{n-2}^{0}\right)=\left(\beta_{n-1}^{0}\right)
$$

Since $\delta d=d \delta$ and also $d \phi^{\sharp}=\phi^{\sharp} d$ one can verify that

$$
\delta\left(\beta_{n-2}^{1}-d\left(\gamma_{n-2}^{0}\right)\right)=0 .
$$


Due to the exactness of the second row we obtain an element $\gamma_{n-3}^{1} \in K_{U}^{n-3,1}$ such that

$$
\delta\left(\gamma_{n-3}^{1}\right)=\beta_{n-2}^{1}-d\left(\gamma_{n-2}^{0}\right) \quad \text { and } \quad d\left(\delta\left(\gamma_{n-3}^{1}\right)\right)=d\left(\beta_{n-2}^{1}\right)=\alpha_{n-2}^{2} .
$$

Repeating this process and inductively verifying the properties at each step, one ends up with the cochain $\gamma_{0}^{n-2} \in K_{U}^{0, n-2}$ such that

$$
\delta\left(\beta_{0}^{n-1}-d\left(\gamma_{0}^{n-2}\right)\right)=0 .
$$

From this one obtains a global $(n-1)$-form $\gamma^{n-1} \in \Omega^{n-1}(X)$, with the property that

$$
\delta\left(\gamma^{n-1}\right)=\beta_{0}^{n-1}-d\left(\gamma_{0}^{n-2}\right) \quad \text { and } \quad d\left(\delta\left(\gamma^{n-1}\right)\right)=\alpha_{0}^{n}=\delta \omega^{n} .
$$

Therefore $d \gamma^{n-1}=\omega^{n}$, which makes $\omega^{n}$ an exact form and the proof complete.

\section{ACKNOWLEDGEMENT}

The author would like to thank the referee for the careful reading of the manuscript, for the suggestion to use the $s$-hyperbolicity condition instead of a curvature bound, and for suggesting the statement of Proposition 3.2 which led to a more elegant proof of Proposition 3.1.

\section{REFERENCES}

[B] A. Borbély, A note on the unclouding the sky of negatively curved manifolds, Bull. Australian Math. Soc. 77 (2008), 413-424. MR2454972

[BO] K. Borsuk, Drei Sätze über die n-dimensionale euklidische Sphäre, Fundamenta Mathematicae 20 (1933), 177-190.

[BS] S. Buyalo, V. Schroeder, Elements of asymptotic geometry, EMS Monographs in Mathematics, European Mathematical Society, Zurich (2007). MR2327160 (2009a:53068)

[BSW] S. Buyalo, V. Schroeder, M. Walz, Geodesics avoiding open subsets in surfaces of negative curvature, Ergodic Theory Dynam. Sys. 20 (2000), 991-1006. MR.1779390(2002e:37045)

[BT] R. Bott, L.W. Tu, Differential forms in algebraic topology, Graduate Texts in Mathematics, 82, Springer-Verlag, New York-Berlin (1986). MR658304(83i:57016)

[LS] L. Lyusternik, S. Shnirel'man, Topological Methods in Variational Methods (Russian), Issledowatelskii Institut Matematiki i Mechanici, Moscow (1930).

[M] Jiri Matoušek, Using the Borsuk-Ulam Theorem, Springer Universitext, 2nd corrected printing, Springer-Verlag, Berlin (2008). MR.1988723 (2004i:55001)

[PP1] J. Parkkonen, F. Paulin, Unclouding the sky of negatively curved manifolds, Geom. and Funct. Anal. 15 (2005), 491-533. MR2153908 (2006i:53048)

[PP2] J. Parkkonen, F. Paulin, Prescribing the behavior of geodesics in negative curvature, arXiv:07062579v1 (2007).

[S] V. Schroeder, Bounded geodesics in manifolds of negative curvature, Math. Z. 235 (2000), 817-828. MR.1801585 (2001m:53065)

[W] M. Walz, Invariant subsets of the geodesic flow on negatively curved manifolds, Thesis, Zurich (1998).

Department of Mathematics and Computer Science, Faculty of Science, Kuwait University, P.O. Box 5969, Safat 13060, Kuwait

E-mail address: borbely.albert@gmail.com 